\title{
Associative Triple Systems with Nondegenerate Bilinear Forms
}

\section{Almotairi KH and Baklouti $A^{*}$}

Umm Al-Qura University, P.O. Box 14035, Makkah, 21955, Saudi Arabia

\begin{abstract}
This paper is bringing a better knowledge of associative triple systems and their related algebraic structures. We prove that any associative triple system is either a $T^{*}$-extension of an associative triple system or an ideal of codimension one of a $\mathrm{T}^{*}$-extension of an associative triple system. Morover, we give several information about the structure of symmetric associative triple systems.
\end{abstract}

Keywords: Associative triple systems; Lie triple systems; $\mathrm{T}^{\star}$ extension

\section{Introduction}

Associative algebras with $\mathrm{n}$-ary compositions in general and associative triple system in particular play important roles in Lie and Jordan theories, geometry, analysis. For instance, associative triple systems give rise to Jordan triple systems [1-3] and Jordan triple systems give rise to 3-graded Lie algebras through the Tists Kantor Koecher construction $[4,5]$, from which most simple Lie algebras can be obtained. Jordan triple systems also give rise to Lie triple systems through the K. Meyberg construction [6,7]. On the other hand, Lie triple systems give rise to graded Lie algebras, which are exactly the kind of Lie algebras associated to symmetric spaces. In geometry and analysis, various types of Jordan triple systems are used in the classifications of different classes of symmetric spaces [2,8-10].

After some preparations, using the notion of $T^{*}$-extention, we will show, in the first section, that there exist a symmetric associative triple systems which is not semisimple. This notion was introduced by Bordemann, who proved that every Jordan algebra of even dimension, which contains an isotropic ideal of dimension $\mathrm{n} / 2$, is a $T^{*}$-extension of a Jordan algebra and every odd dimensional Jordan algebra is an ideal of codimension one of a $T^{*}$-extension. Lin et al. extended this notion to Lie triple systems. We will show that the same theorem holds for associative triple systems. The proof in this case is different from the Jordan algebra case. It relies the construction of Lie triple systems associated to an associative one.

\section{Definition 1}

An assocaitive triple system is a vector space $A$ over a field $K$ with a trilinear multiplication $\{a, b, c\}$ satisfying

$$
\{\{a, b, c\} d, e\}=\{a,\{b, c, d\}, e\}=\{a, b\{c, d, e\}\},
$$

for any $a, b, c, d, e \in A$.

\section{Definition 2}

A derivation of an associative triple system $\mathrm{A}$ is a linear transformation $\mathrm{D}$ of $\mathrm{A}$ into A such that

$$
D(\{a, b, c\})=\{D(a), b, c\}+\{a, D(b), c\}+\{a, b, D(c)\}, \forall a, b, c \in \mathrm{V}
$$

The set of all derivation of $\mathrm{V}$ is denoted by $\operatorname{Der}(A)$. The set $\operatorname{Der}(A)$ of derivations of $A$ is a Lie algebra of linear transformations, we call it the derivation algebra of $A$. Further, if $a, b \in A$, the linear maps $L(a, b)$ (resp. $R(a, b)$ ) defined on $A$ by $L(a, b) c=(a, b, c)$ (resp. $R(a, b) c=(c, a, b)), \forall c \in A$, is a derivation of $A$. The linear maps $\mathrm{L}(a, b)$ (resp. $R(a, b))$ are called the left (resp.right) multiplications of $A$. $L(T, T)=\left\{\sum_{a_{i}, b_{i}} L\left(a_{i}, b_{i}\right) ; a_{i}, b_{i} \in A\right\}$ is a subalgebra of $\operatorname{Der}(A)$, we call it we call it the inner derivation algebra of $A$, its element is called an inner derivation of $A$.

\section{Definition 3}

(1) A symmetric bilinear form B on an asoociative triple system A is called to be invariant if it is right and left invariant. That is

$$
\omega(a,\{d, c, b\})-\omega(b,\{d, c, a\})+\omega(c,\{b, a, d\})+\omega(d,\{a, b, c\})=0,
$$

It was proved [11] that if $B$ is a symmetric and right invariant bilinear form on $A$, then $B$ is left invariant. Therefore, a symmetric bilinear form $B$ is invariant if and only if it is right invariant.

(2) We say that $(A, B)$ is a symmetric associative triple system if $B$ is a non-degenerate symmetric invariant bilinear form on $A$. Here $B$ is called a symmetric structure on $A$. A symmetric associative triple system $(A, B)$ is said to be reducible (or B-reducible) if it admits an ideal $J$ such that the restriction of $B$ to $J \times J$ is non-degenerate. Otherwise, we will say $(T, B)$ is irreducible.

(3)We say that $(A, \omega)$ is a symplectic associative triple system if $\omega$ is a non-degenerate skew-symmetric bilinear form on $\mathrm{T}$ such that the identity.

$$
\omega(a,\{d, c, b\})-\omega(b,\{d, c, a\})+\omega(c,\{b, a, d\})+\omega(d,\{a, b, c\})=0,
$$

holds for any $a, b, c, d \in A$. Here $B$ is called a symplectic structure on $A$.

\section{Definition 4}

An element $f \in \operatorname{Hom}(A, A)$ is called $B$-symmetric, (resp. $B$ antisymmetric) if $B(f(a), b)=B(a, f(b)),($ resp. $B(f(a), b)=-B(a, f(b)), \forall a, b \in A$. Denote by $\operatorname{Hom}_{s}(A, A)$ (resp.Hom $(A, A)$ the subspace of $B$-symmetric (resp.B-antisymmetric) endomorphism of $A$.

Theorem 1: A symmetric associative triple system $(A, B)$ admits a symplectic form $\omega$, if and only if there exists a $B$-antisymmetric invertible derivation $\delta$ of $A$ such that $\omega(a, b)=B((\mathrm{a}), \mathrm{b}), \forall a, b \in A$.

Proof. Since $B$ and $\omega$ are two nondegenerate bilinear forms, there

${ }^{*}$ Corresponding author: Baklouti A, Umm Al-Qura University, P.O. Box 14035 Makkah, 21955, Saudi Arabia, Tel: +966-12-527-0000; E-mail: ambaklouti@uqu.edu.sa

Received October 12, 2017; Accepted November 15, 2017; Published November 21, 2017

Citation: Almotairi KH, Baklouti A (2017) Associative Triple Systems with Nondegenerate Bilinear Forms. J Phys Math 8: 254. doi: 10.4172/20900902.1000254

Copyright: (c) 2017 Almotairi $\mathrm{KH}$, et al. This is an open-access article distributed under the terms of the Creative Commons Attribution License, which permits unrestricted use, distribution, and reproduction in any medium, provided the original author and source are credited. 
exists $\delta \in \operatorname{Hom}(A, A)$, such that $\omega(a, b)=B((a), b), a, b \in A$. Further, since $\omega$ is symplectic, then;

$B(\delta(\{a, b, c\}), d)=B(\{\delta(a), b, c)\}, d)+B(\{a, \delta(b), c)\}, d)+B(\{a, b, \delta(c))\}, d), \forall a, b, c, d \in A$.

The fact that $B$ is nondegenerate implies that is a derivation of $A$. Conversely, if $\delta$ is a $B$-antisymmetric invertible derivation of $A$, then it is clear that the bilinear form $\omega: A \times A \mathrm{~K}$ defined by: $\omega(a, b)=B((a), b), a, b \in A$, is a symplectic form of $A$.

Theorem 2: Let $(A,\{\}$,$) be a asoociative triple system and \Theta$ : $A \times A \times A \rightarrow A^{*}$ be a trilinear map. Let $T_{\Theta}^{*}(A)=A \oplus A^{*}$ on which it is defined a symmetric bilinear distributive triple product

$\{a+f, b+g, c+h\}=\{a, b, c\}_{J}+\Theta(a, b, c)+\{f, b, c\}+\{a, g, c\}+\{a, b, h\}$,

For all $a, b, c \in A$ and $f, g, h \in A^{*}$. With,

$\{f, b, c\}(d)=f(\{d, c, b\}) ;\{a, g, c\}(d)=g(\{a, d, c\})$ and $\{a, b, h\}(d)=h(\{b, a, d\})$

is an asoociative triple system if and only if, $\Theta$ satisfies

$\Theta(a, b,\{c, t, r\})+\{a, b, \Theta(c, t, r)\}=\Theta(\{a, b, c\}, t, r)+$

$\{\Theta(a, b, c), t, r\}=\{a, \Theta(b, c, t), r\}+\Theta(a,\{b, c, t), r)$,

$\forall a, b, c, t, r \in A$. Furthermore, the bilinear form $B$ defined on $T_{\Theta}^{*}(A)$ by:

$B(a+f, b+g)=g(a)+f(b), \forall a, b \in D, f, g \in D^{*}$

is dn invdridnt scalar product on $T_{\Theta}^{*}(A)$ if and only if $\Theta$ satisfies

$\Theta(a, b, c)(d)=\Theta(b, a, d)(c), \forall a, b, c, d \in A$.

The constructed symmetric asoocidtive triple system $\left(T_{\Theta}^{*}(A), B\right)$ is called the $T_{\Theta}^{*}$-extension of $A$ by means of $\Theta$.

Proof. Computation [12,13].

Theorem 3: Let $A$ be a associative triple system which admits an invertible derivation $D$ and a trilinear map : $A \times A \times A A^{*}$ satisfying (2) and (3). Then the $T^{*}$-extension $T_{\Theta}^{*}(A)$ of $A$ admits a symplectic structure.

Let $\tilde{D} \in \operatorname{Hom}\left(T_{\Theta}^{*}(A), T_{\Theta}^{*}(A)\right)$ defined by

$\tilde{D}(a+f):=D(x)-f \circ D, \forall a \in A$ and $f \in A^{*}$.

$B(\tilde{D}(a+f), b+g)=-f(b)+g(a)=-B(a+f, \tilde{D}(b+g))$. Thus $\tilde{D}$ is an invertible $B$-skew symmetric derivation of $T_{\Theta}^{*}(A)$. We get the result by Theorem 0.1 .

Example 1: Let $A$ be an associatif triple system and $n \in \mathrm{N} ; n>1$. Consider the non-unitary associative algebra $F_{n}=t \mathrm{~K}[t] / t^{n} \mathrm{~K}[t]$. Define the bracket on the vector space $A_{n}=T \otimes F_{n}$ by

$\left\{x \otimes t^{p}, y \otimes t^{q}, z \otimes t^{r}\right\}=\{x, y, z\} \otimes t^{p+q+r}$,

where $x, y, z \in A$ and $p, q, r \in \mathbb{N} \neq\{0\}$. Then $A_{n}$ is a nilpotent associative triple system. The endomorphism $D$ of $A_{n}$ defined by

$$
D\left(x \otimes t^{p}\right)=p\left(x \otimes t^{p}\right)
$$

for any $x A$ and $p\{1, \ldots, n-1\}$ is an invertible derivation of $A_{n}$. Define the product on the vector space $\tilde{T}_{n}=T_{n} \oplus\left(T_{n}\right)^{*}$ by

$$
\{x+f, y+g, z+h\}=\{x, y, z\}_{A_{n}}+\{f, y, z\}+\{x, g, z\}+\{x, y, h\},
$$

With,

$\{f, y, z\}(a)=f(\{a, z, y\}) ;\{x, g, z\}(a)=g(\{x, a, z\})$ and $\{x, y, h\}(a)=h(\{y, x, a\})$,

for all $x, y, z, a \in A_{n}$ and $f, g, h \in A_{n}^{*}$.

Define the bilinear form on $T_{n}$ by $B(X+f, Y+g)=f(Y)+g(X)$. Then $\left(\tilde{T}_{n}, B\right)$ is a symmetric associative triple system. Define $\tilde{D}$ on $\tilde{T}_{n}$ by
$\tilde{D}(X+f)=D(X)-f \circ D$. Then $\tilde{D}$ is an invertible derivation which is skew-symmetric with respect to $B$. Hence, the symmetric associative triple system $\left(T_{n}, B\right)$ admits a symplectic structure.

Proposition 1: Let $\mathrm{V}$ be an associative triple system and $Z(V)=\{a \mathrm{~V} ;\{a, b, c\}=0, \forall b, c \in \mathrm{V}\}$ be the center of $\mathrm{V}$. Then, $(\mathrm{Z}(\mathrm{V}))^{\perp}=(\mathrm{V}, \mathrm{V}, \mathrm{V})$, where $(\mathrm{V}, \mathrm{V}, \mathrm{V})$ is the sub-space of $V$ spanned by the set $\{\{a, b, c\} ; a, b, c \in \mathrm{V}\}$.

Let $a, b, c \in \mathrm{V}$ and let $x Z(\mathrm{~V})$. Then, $\mathrm{B}(\{a, b, c\}, x)=B(a,\{a, b, c\})=0$. So, $\{\mathrm{V}, \mathrm{V}, \mathrm{V}\} \subseteq(\mathrm{Z}(\mathrm{V}))^{\perp}$. Conversely, let $\mathrm{y} \in(\mathrm{V}, \mathrm{V}, \mathrm{V})^{\perp}$. Using the invariance of $B$ we get, $B((y, b, a), c)=0$, for $c \in \mathrm{V}$. Thus, $(y, b, a)=0$, for $a, b \in \mathrm{V}$ because $B$ is nondegenerate. Hence, $y \in Z(V)$. Consequently, $(\mathrm{Z}(\mathrm{V}))^{\perp}=\{\mathrm{V}, \mathrm{V}, \mathrm{V}\}$.

\section{Definition 5}

Let $(\mathrm{V},()$,$) be an associative triple system. We define the descending$ series $\left(\mathrm{V}^{n}\right)_{n \in \mathrm{N}}$ by $\mathrm{V}^{0}=\mathrm{V}$ and $\mathrm{V}^{n+1}=\left(\mathrm{V}^{n}, \mathrm{~V}^{n}, \mathrm{~V}\right), \forall n \in \mathrm{N}$ and the ascending series $\left(\mathrm{V}^{(n)}\right)_{n \in \mathrm{N}}$ by $\mathrm{V}^{(0)}=A$ and $\mathrm{V}^{(n+1)}=\left(\mathrm{V}^{(n)}, \mathrm{V}^{(n)}, \mathrm{V}^{(n)}\right), \forall n \mathrm{~N}$. If there exists $n \in \mathrm{N}$ such that $\mathrm{V}^{n}=\{0\}\left(\right.$ resp. $\left.\mathrm{V}^{(n)}=\{0\}\right)$, then $A$ is called solvable (resp. nilpotent).

\section{Definition 6}

Let $\mathrm{V}$ be an associative triple system and $B$ be an invariant scalar product on $V$.

1. An ideal $\mathrm{U}$ of $\mathrm{V}$ is a subspace of $A$ which satisfies $(\mathrm{U}, \mathrm{V}, \mathrm{V})+(\mathrm{V}, \mathrm{U}, \mathrm{V}) \subseteq \mathrm{U}$.

2. An ideal $U$ of $\mathrm{V}$ is said to be:

(a) Abelian if $\{U, U, U\}=\{0\}$.

(b) Solvable (resp. nipotent) if it is solvable (resp. nilpotent) as a asoociative triple system.

(c) Nondegenerate (resp. degenerate) if $B_{\mid \mathrm{U} \times \mathrm{U}}$ is nondegenerate (resp. degenerate).

3. The largest solvable ideal of $V$ is called the radical of $V$ and denoted $\operatorname{Rad}(V)$.

4. The asoociative triple system $(\mathrm{V}, B)$ is called.

(a) Semi-simple if it has no non trivial solvable ideal. That is $\operatorname{Rad}(V)=\{0\}$.

(b) B-irreducible, if $\mathrm{V}$ contains no non-trivial nondegenerate ideal.

The following lemma is straightforward.

Lemma 1: Let $(\mathrm{V}, \mathrm{B})$ be an associative triple system and $U$ be an ideal of $\mathrm{V}$. Then,

$\mathrm{U}^{\perp}=\{x \in \mathrm{V}, B(x, y)=0 \forall y \in A\}$ is an ideal of $\mathrm{V}$.

If $\mathrm{U}$ is nondegenerate, then $A=\mathrm{U} \oplus \mathrm{U}^{\perp}$ and $\mathrm{U}^{\perp}$ is also nondegenerate.

Lemma 2: Let $(\mathrm{V}, \mathrm{B})$ be an associative triple system. Then, $\mathcal{V}=\bigoplus_{i=1}^{r} \mathcal{V}_{i}$ where $r \in N$ and such that for $i \in\{1, \ldots, r\}$,

$\mathrm{V}_{i}$ is a nondegenerate ideal of $\mathrm{V}$.

$\mathrm{V}_{i}$ is $B$-irreducible as an associative triple system.

For $i \neq j$ and $(x, y) \in \mathrm{V}_{i} \times \mathrm{V}_{j}$, we have $B(x, y)=0$.

We precede by induction on $n=\operatorname{dim}(\mathrm{V})$. If $n=1$, then the assertion is true. Suppose that every asoociative triple system of dimension less than $n$ satisfies the proposition. Let $(V, B)$ be an associative triple system of dimension $n+1$. If $\mathrm{V}$ does not contain any non trivial nondegenerate 
ideal, then the assertion is true for $\mathrm{r}=1$. If not, let $\mathrm{I}$ be a non trivial nondegenerate ideal of $\mathrm{V}$. By the Lemma $1, \mathrm{~V}=\mathrm{I} \oplus \mathrm{I}^{\perp}$. The result follows by applying the induction to I and $\mathrm{I}^{\perp}$. W.

Proposition 2: Let $(V, B)$ be a semi-simple asoociative triple system and consider the decomposition $\mathcal{V}=\oplus_{i=1}^{r} \mathcal{V}_{i}$ of $V$ as in the Lemma 2.

If $\mathrm{I}$ is a simple ideal of $\mathrm{V}$, then there exists $i_{0} \in\{1, \ldots, r\}$ such that $\mathcal{V}_{i_{0}}=\mathcal{I}$

For $i \in\{1, \ldots, r\}, \mathrm{V}_{i}$ is simple.

(i) Let I be a non-trivial simple ideal of V. Assume that for all $i \in\{1, \ldots, r\}$ we have $\mathrm{I} \cap \mathrm{V}_{i}=\{0\}$. Since $\{\mathcal{I}, \mathcal{V}, \mathcal{V}\} \subseteq \bigoplus_{i=1}^{r}\left(\mathcal{I} \cap \mathcal{V}_{i}\right)=0$. Then, $\{\mathrm{I}, \mathrm{I}, \mathrm{I}\}=0$ and $\mathrm{I}$ is solvable. Hence, there exists $i_{0} \in\{1, \ldots, r\}$ such that $\mathcal{I} \cap \mathcal{V}_{i_{0}} \neq\{0\}$. Since $\mathcal{I} \cap \mathcal{V}_{i_{0}}$ is an ideal of $I$ and $I$ is simple, then $\mathcal{I} \cap \mathcal{V}_{i_{0}}=\mathcal{I}$. So, $\mathcal{I} \subseteq \mathcal{V}_{i_{0}}$. The fact that $\mathcal{V}_{i_{0}}$ is $B$-irreducible and I is nondegenerate, imps that $\mathcal{I}=\mathcal{V}_{i_{0}}$. (ii) Suppose that there exists $i \in\{1, \ldots, r\}$ such that $\mathrm{V}_{i}$ is not simple. Then, without lost of generality, we may write $\mathcal{V}=\left(\oplus_{i=1}^{s} \mathcal{V}_{i}\right) \oplus\left(\oplus_{i=s+1}^{r} \mathcal{V}_{i}\right)$ where for $1 \leq i s, V_{i}$ is simple and $\mathrm{V}_{i}$ is not simple for $s+1 \leq I r$. Since $\mathrm{V}$ is semi-simple, then we can consider the decomposition $\mathcal{V}=\bigoplus_{i=1}^{l} \mathcal{V}_{i}$ of $\mathrm{V}$ into the direct sum of its simple ideals. The assertion (i) imps that $s=l=r$, W.

The previous Proposition shows that, in the case of semisimples triples systems, the decompsition into the direct sum of orthogonal nondegenerate ideals coincides with the decomposition into a direct sum of simple ideals.

The following theorem presents a process of construction of a symmetric asoociative triple systems.

Remark 1: It is clear that $J^{*}$ is an abelian ideal of $T^{*}{ }_{w} J$. Thus, $T^{*}{ }_{w} J$ is not semi-simple. Moreover, if $J$ is not nilpotent, then $T^{*}{ }_{w} J$ is not nilpotent too. Consequently, the family of a symmetric asoociative triple systems contains strictly the of semi-simple asoociative triple systems and the symmetric nilpotent asoociative triple systems.

Theorem 4: Let $(\mathrm{J}, \mathrm{B})$ be a a symmetric asoociative triple system of dimension $n$. Then, $(J, B)$ is isometric to a $T^{*}$-extension $\left(T_{w}^{*}\left(\mathfrak{J}_{1}\right), B_{1}\right)$ if and only if $\mathrm{n}$ is even and $J$ contains an isotropic ideal I of dimension $n / 2$.

Let $\mathrm{I}$ be an isotropic ideal of $\mathrm{J}$ of dimension $\mathrm{n} / 2$. Since $B$ is nondegenerate, the ideal $\mathrm{I}$ is abelian. Let us consider $\mathrm{V}$ an isotropic complementary to I. Then, $\mathrm{J}=\mathrm{I} \oplus \mathrm{V}$ and $\mathrm{V}^{\perp}=\mathrm{V}$. Let $x, y, w \in \mathrm{V}$. Put $\{x, y, z\}=(x, y, z)+\beta(x, y, z)$ where $\alpha(x, y, z) \in \mathrm{I}$ and $\beta(x, y, z) \in \mathrm{V}$. It is easy to check that $(\mathrm{V}, \beta)$ is a asoociative triple system.

Now, since $B$ is nondegenerate, the linear map $v: \mathrm{I} \rightarrow \mathrm{V}^{*} ; i \rightarrow B(I,$. is invertible. Furthermore, $\operatorname{dim}(\mathrm{I})=\mathrm{n} / 2=\operatorname{dim}\left(\mathrm{V}^{*}\right)$. Thus, $v$ is an isomorphism of vector spaces. We consider the $T^{*}$-extension $T_{w}^{*}(\mathcal{V})=\mathcal{V} \oplus \mathcal{V}^{*}$ of $\mathrm{V}$ by means of the trilinear map $\omega: \mathrm{V} \times \mathrm{V} \times \mathrm{V} \rightarrow \mathrm{V}^{*}$ defined by $w(x, y, z)=v((x, y, z)), x, y, z \in \mathrm{V}$. Moreover, let us consider $\Omega: \mathfrak{J}=\mathcal{I} \oplus \mathcal{V} \rightarrow \mathcal{V} \oplus \mathcal{V}^{*} ; i+x \mapsto x+v(i)$

Using the invariance of the bilinear form $B$ on $J$, we get for all $x, y, z \in \mathrm{V}, i \in \mathrm{I}$,

$v(i)(\{x, y, z\})=B(i,\{x, y, z\})=B(\{i, z, y\}, x)=v(\{i, z, y\})(x)$.

Similarly,

$$
v(i)(\{x, y, z\})=v(\{z, i, x\})(y)=v(\{y, x, i\})(z) .
$$

Consequently, if $\Omega: \mathrm{J}=\mathrm{IV} \rightarrow \mathrm{VV}^{*}$ défined by $i+x \mapsto x+v(i), \forall i \in \mathcal{I}, x \in \mathcal{V}$ then, for $X=i+x, Y=j+y, Z=k+z \in \mathrm{J}=\mathrm{IV}$, we get

$$
\begin{aligned}
& \Omega(\{X, Y, Z\})=\beta(x, y, z) \oplus v(\alpha(x, y, z))+v(\{x, y, k\})+ \\
& v(\{i, y, z\})+v(\{x, j, z\}) 1,6 \mathrm{~cm} \\
& =\beta(x, y, z) \oplus w(x, y, z)+v(k)(\{y, x, .\})+v(i)(\{., z, y\})+ \\
& v(j)(\{z, ., x\}) 1,6 \mathrm{~cm}=\{\Omega(X), \Omega(Y), \Omega(Z)\} .
\end{aligned}
$$

Thus, $\Omega$ is an isomorphism of asoociative triple systems $\mathrm{W}$.

Theorem 5: Let $(\mathrm{J}, B)$ be a a symmetric asoociative triple system of dimension $n$. If $n$ is odd,and I is an isotropic ideal of $J$ of dimension $[\mathrm{n} / 2]$. Then, $J$ is isomorphic to a nondegenerate ideal of codimension 1 in a $T^{*}$-extension of the asoociative triple system $\mathrm{J} / \mathrm{I}$.

Let I be an isotropic ideal of $\mathrm{J}$ of dimension [n/2] and let $L(\mathrm{~J})$ be the asoociative triple system generated by the left, right and middle multiplications of J. Since $B$ is invariant on J. By Lemma of [?], $\phi\left(\mathrm{I}^{\perp}\right) \subseteq \mathrm{I}$, for all $\phi \in L(J)$. Consequently,

$$
\left\{\mathfrak{J}, \mathfrak{J}, \mathcal{I}^{\perp}\right\}+\left\{\mathfrak{J}, \mathcal{I}^{\perp}, \mathfrak{J}\right\}+\left\{\mathcal{I}^{\perp}, \mathfrak{J}, \mathfrak{J}\right\} \subseteq \mathcal{I} .
$$

So,

$$
\mathcal{I}^{\perp} \subseteq\left(\left\{\mathfrak{J}, \mathfrak{J}, \mathcal{I}^{\perp}\right\}+\left\{\mathfrak{J}, \mathcal{I}^{\perp}, \mathfrak{J}\right\}+\left\{\mathcal{I}^{\perp}, \mathfrak{J}, \mathfrak{J}\right\}\right)^{\perp} .
$$

Since $B$ is invariant and nondegenerate, then $\left\{\mathrm{J}, \mathrm{J}, \mathrm{I}^{\perp}\right\}+\left\{\mathrm{J}, \mathrm{I}^{\perp}, \mathrm{J}\right\}+\left\{\mathrm{I}^{\perp}, \mathrm{J}, \mathrm{J}\right\}=\{0\}$. Therefore, $\mathrm{I}^{\perp}$ is abelian. Now, let us consider the one dimensional abelian asoociative triple system $\mathrm{Kc}$ endowed with the bilinear form $B_{c}: \mathrm{K} c \times \mathrm{K} c \rightarrow \mathrm{K}$ defined by $B_{c}(c, c)=1$. Let $\mathrm{J}_{1}=\mathrm{J} \oplus \mathrm{K} c$ be the asoociative triple system endowed with the triple product given by:

$$
\{x+\alpha c, y+\gamma c, z+\lambda c\}=\{x, y, z\}, \forall x, y, z \in \mathfrak{J}, \alpha, \gamma, \lambda \in \mathbb{K} .
$$

We define on $\mathrm{J}_{1}$ the bilinear form $\mathrm{B}_{1}$ by:

$$
B_{1_{\mathfrak{J} \times \mathfrak{J}}}=B, B_{1}(c, c)=1 \text {, and } B_{1}(\mathfrak{J}, c)=B_{1}(c, \mathfrak{J})=0 \text {. }
$$

It is clear that $\left(\mathrm{J}_{1}, B_{1}\right)$ is a pseudo Euclidean asoociative triple system. Besides, $\mathrm{J}_{1}$ is a nondegenerate ideal of codimension 1 of $\mathrm{J}_{1}$. Let $d \in \mathrm{I}^{\perp}$ such that $B(d, d)=-1$ and consider $\mathrm{I}_{1}=\mathrm{IK} e$, where $e=c+d$. It is easy to see that $\mathrm{I}_{1}$ is an ideal of $\mathrm{J}_{1}$. In addition,

$B_{1}(e, e)=B(d, d)+B_{c}(c, c)=0$, and $B_{1}(x, e)=B(x, d)+B(x, c)=0, \forall x \in \mathcal{I}$.

So, $\mathrm{I}_{1}$ is isotropic and, $\operatorname{dim}\left(\mathcal{I}_{1}\right)=\frac{n+1}{2}$. Since dimension of $\mathrm{J}_{1}$ is even, then the theorem 4 imps that $J_{1}$ is isomorphic to a $T^{*}$-extension of the asoociative triple system $J_{1} / I_{1}$.

Cosequently, $\mathrm{J}_{1} / \mathrm{I}_{1}$ is isomorphic to $\mathrm{J} / \mathrm{I}, \mathrm{W}$.

\section{Acknowledgements}

The authors would like to thank Deanship of Scientific Research at Umm AlQura University (Project ID 15-SCI-3-1-0025) for the financial support.

\section{References}

1. Jacobson N (1949) Lie and Jordan triple systems. Amer J Math 71: 149-170.

2. Lister WG (1952) A structure theory of lie triple systems. Trans Amer Math Soc 72: $217-242$

3. Neher E (1985) On the classification of lie and Jordan triple systems. Comm Alg 13: 2615-2667.

4. Kantor IL (1964) Classification of irreducible transitive differential groups. Dok Akad Nauk SSSR 158: 1271-1274.

5. Koecher M (1967) Imbedding of Jordan algebras into lie algebras. I Amer J Math 89: 787-816

6. Gaunaydin M, Hyun S (1991) Ternary algebraic construction of extended superconformal algebras. Modern Phys Lett A 6: 1733-1743.

7. Gaunaydin M, Hyun S (1992) Ternary algebraic approach to extended superconformal algebras. Nuclear Phys B 373: 688-712. 
Citation: Almotairi KH, Baklouti A (2017) Associative Triple Systems with Nondegenerate Bilinear Forms. J Phys Math 8: 254. doi: 10.4172/20900902.1000254

Page 4 of 4

8. Bertram W (2000) The geometry of Jordan and lie structures. Lectures Notes in Math, Springer, Berlin.

9. Chu C-H (2008) Jordan triples and Riemannian symmetric spaces. Adv Math 219: 2029-2057.

10. Kaup W (1983) A Riemann mapping theorem for bounded symmetric domains in complex Banach spaces. Math Z 183: 503-529.
11. Zhang ZX, Shi YQ, Zhao LN (2002) Invariant symmetric bilinear forms on Lie triple systems. Comm Algebra 30: 5563-5573.

12. Baklouti A (2017) Quadratic Hom-Lie triple systems. J Geom Phys 121: 166175

13. Baklouti A, Benayadi S (2015) Pseudo-Euclidean Jordan algebras. Comm Alg 43: 2094-2123. 